\title{
Caracterização demográfica e morfométrica das síndromes hipertensivas da gestação
}

\section{Demographic and morphometric characterization of the Gestational Hypertensives Syndromes.}

\section{Caracterización demográfica y morfométrica de los síndromes hipertensivos del embarazo.}

Larissa Faquim Bazaga', Sanívia Aparecida de Lima Pereira"', Renata Calciolari Rossi"',

Camila Lourencini Cavellanilv, Camila Souza de Oliveira Guimarães ${ }^{\vee}$, Ana Karina Marques Salge ${ }^{\mathrm{V} \text {, }}$

Vicente de Paula Antunes Teixeira ${ }^{V I I}$, Eumenia Castro da Cunha Castro ${ }^{I I I}$, Rosana Rosa Miranda Corrêa ${ }^{I X}$

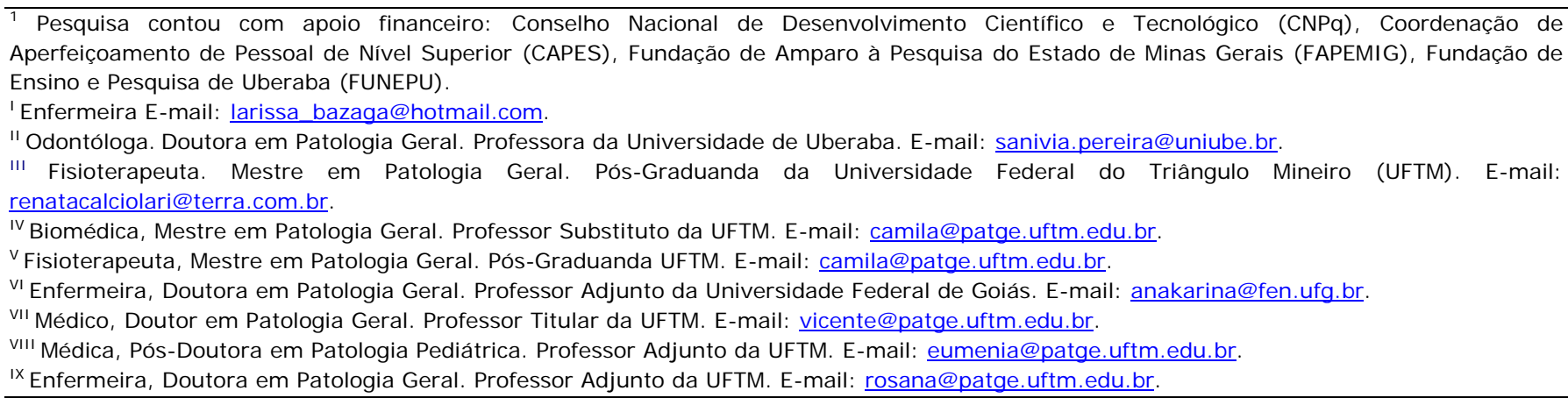

\section{RESUMO}

As Síndromes Hipertensivas da Gestação (SHG) possuem intensidade variável de acordo com seu tipo. Com este estudo objetivou-se descrever as características demográficas materno- fetais e as alterações morfométricas placentárias nos diferentes tipos de SHG, pela análise retrospectiva dos prontuários de pacientes hipertensas, com placentas avaliadas morfologicamente, processadas para imunohistoquímica utilizando anticorpo monoclonal anti-human CD31 e analisadas morfometricamente. Os resultados demonstraram que, entre as SHG, os grupos com Hipertensão Crônica (HC) e Préeclâmpsia sobreposta à Hipertensão Crônica (PSHC) apresentaram idade materna superior $(p=0,017)$. A freqüência das SHG foi superior entre as multíparas. Menor idade gestacional, menores índices de Apgar e pesos placentários inferiores ao esperado para a população $(p<0,05)$ foram observados nos casos com Pré-eclâmpsia (PE) e PSHC. Não houve diferença no número de vasos das vilosidades tronco placentárias. Nas demais, houve aumento nos casos com PE e Hipertensão Gestacional (HGE) e redução nos casos com PSHC. A importância do estudo consiste na descrição demográfica e morfológica nas SHG, visando melhorar o atendimento obstétrico e a compreensão dos diferentes tipos de SHG. A gravidade do quadro clínico das SHG tem relação com maiores intercorrências materno-fetais e com alterações no padrão vascular placentário.

Descritores: Gravidez; Hipertensão; Morfologia; Placenta.

\section{ABSTRACT}

The hypertensive disorders of pregnancy (SHG) have variable intensity according to their type. This study aimed to describe the demographic characteristics and maternal-fetal morphometric changes of placenta in different types of SHG by retrospective analysis of medical records of hypertensive patients with placentas evaluated morphologically, processed for immunohistochemistry using monoclonal antibody anti-human CD31 and analyzed morphometrically. The results showed that among the SHG, the groups with chronic hypertension $(\mathrm{CH})$ and Pre-eclampsia superimposed on chronic hypertension (PSHC) showed higher maternal age $(p=0017)$. The frequency of the SHG was higher among multiparous. Lower gestational age, lower Apgar score and placental weights lower than expected for the population $(p<0.05)$ were observed in cases with pre-eclampsia (PE) and PSHC. There was no difference in the number of vessels of placental stem villi. In the others, there was an increase in cases with PE and gestational hypertension (HGE) and reduction in cases with PSHC. The importance of the study is to describe the demographic and morphological SHG to improve the obstetric care and understanding of different types of SHG. The severity of symptoms of SHG is related to higher maternal and fetal complications and changes in placental vascular patern.

Descriptors: Pregnancy; Hypertension; Morphologic; Placenta.

\section{RESUMEN}

Los Síndromes Hipertensivos del Embarazo (SHG) tienen una intensidad variable de acuerdo con su tipo. El objetivo de este etudio fue describir las características demográficas materno-fetales y alteraciones morfométricas placentarias entre diferentes tipos de SHG. Los resultados mostraron que, entre los SHG, los grupos con Hipertensión Crónica (HC) e Preeclampsia relacionada a HC (PSHC) presentaron una edad materna estadísticamente superior $(p=0,017)$. La frecuencia de los SHG fue superior entre las pacientes multíparas. Fue observada menor edad gestacional, menores índices de Apgar y pesos placentarios inferiores a los esperados para la populación general $(p<0,05)$ en los casos con Preeclampsia (PE) y PSHC. No hubo diferencia en el número de vasos del tronco de las vellosidades placentarias. En los demás, hubo aumento en los casos con PE e Hipertensión Gestacional (HGE) y reducción en los casos con PSHC. La importancia del estudio consiste en la descripción demográfica y morfológica de los SHG, promoviendo mejorar el atendimiento obstétrico y la comprensión de los diferentes tipos de SHG. La gravedad del cuadro clínico de los SHG está relacionada con mayores complicaciones maternofetales y con alteraciones en el padrón vascular placentario.

Descriptores: Embarazo; Hipertensión; Morfología; Placenta. 


\section{NTRODUÇÃO}

A placenta é considerada uma fonte fidedigna de informações em relação à origem de dados relevantes sobre a fisiopatogenia de intercorrências perinatais ${ }^{(1)}$. Alterações morfológicas placentárias podem indicar doenças maternas sistêmicas como a hipertensão, diabetes e infecções e intercorrências fetais, como crianças pequenas para a idade gestacional $^{(2)}$. Uma ampla variedade de alterações morfológicas é descrita nas placentas de pacientes com pré-eclâmpsia $(\mathrm{PE})$, entretanto, a patogênese definitiva destas alterações estruturais e sua relação com a função placentária relacionada à perda perinatal e morbidade em gestações de alto risco permanecem desconhecidas ${ }^{(3)}$.

As Síndromes Hipertensivas da Gestação (SHG) são uma das principais complicações gestacionais sendo responsáveis por alto índice de morbidade e mortalidade entre os recém-nascidos ${ }^{(4)}$. As alterações maternas compreendem a alterações endoteliais difusas, de intensidade variável de acordo com o tipo de $\mathrm{SHG}^{(5)}$. Entre as alterações perinatais, há aumento da frequência de prematuridade, da restrição do crescimento intra-uterino (RCIU) e da morbimortalidade devido a hipóxia intra-uterina, alterações que possuem grande repercussão na assistência materno-fetal ${ }^{(6)}$.

$\mathrm{Na}$ PE há falha de adaptação das arteríolas deciduais maternas, dificultando a transferência de sangue para o espaço interviloso e a troca de nutrientes entre a circulação materna e fetal ${ }^{(7)}$, provocando proliferação compensatória dos vasos placentários. A hipóxia uteroplacentária é responsável por alterações morfológicas como aumento da angiogênese e proliferação trofoblástica, produzindo vilosidades terminais altamente vascularizadas $^{(8)}$, o que compensa a perda do tecido viloso e mantém a capacidade de troca ${ }^{(9)}$.

Embora muitos trabalhos enfatizem intervenções clínicas e alterações morfológicas da PE, não foram encontrados na literatura estudos que avaliassem as diferenças desses parâmetros em cada tipo de SHG. Portanto, o presente estudo tem como objetivo descrever as características demográficas maternas e fetais e as alterações na vascularização placentária de pacientes com os diversos tipos de SHG.

\section{METODOLOGI A}

Após aprovação pelo Comitê de Ética da UFTM (protocolo 350), foram avaliados retrospectivamente os prontuários de partos realizados no Hospital Escola da Universidade Federal do Triângulo Mineiro (UFTM), Uberaba, Minas Gerais, no período de 2000 a 2002, cujas placentas já tinham sido previamente examinadas na Disciplina de Patologia Geral da UFTM. A revisão dos prontuários de todas as pacientes com placentas examinadas neste período teve como objetivo coletar informações relativas à idade e a história obstétrica materna. Os pesos das crianças foram comparados aos de estudos populacionais, de acordo com os valores estabelecidos para idade gestacional ${ }^{(10)}$. Foram incluídos todos os casos de gestantes que apresentaram SHG, de acordo com os critérios clínicos e laboratoriais, sendo divididas em grupos: Hipertensão Gestacional, Hipertensão Crônica, Préeclâmpsia, Pré-eclâmpsia sobreposta à Hipertensão crônica ${ }^{(5,11)}$ e Não Hipertensas $(\mathrm{NH})$. Foram excluídas pacientes com doenças que poderiam comprometer o fluxo placentário, como doenças vasculares maternas diferentes de hipertensão arterial, doença autoimune e diabetes. O grupo de pacientes $\mathrm{NH}$ foi composto por casos de gestantes clinicamente normais, com recém-nascidos sem intercorrências e ausência de alterações na morfologia placentária. Após os critérios de inclusão e exclusão, foram selecionados 91 casos. Os fragmentos placentários foram processados para histoquímica e imunohistoquímica. Posteriormente foi realizada a quantificação dos vasos placentários nas lâminas coradas pela Hematoxilina e Eosina, sendo avaliados os vasos das vilosidades terminais em três áreas distintas: na camada logo abaixo do prato coriônico, na placa basal e entre estas duas áreas, totalizando em 60 por área. Para caracterização da proliferação e distinção vascular nos casos com edema intravilositário foram selecionadas 30 placentas pareadas pela idade gestacional de todos os grupos de SHG, processadas para a imunohistoquímica com 0 anticorpo monoclonal anti-human CD31 (PECAM-1). Foram consideradas estatisticamente significativas as diferenças em que a probabilidade $(p)$ foi menor que $5 \%(p<0,05)$.

\section{RESULTADOS E DISCUSSÃO}

Das 91 placentas analisadas, 18 (19,8\%) pertenciam ao grupo $\mathrm{NH}, 6(6,6 \%)$ ao grupo $\mathrm{HC}, 8$ $(8,8 \%)$ ao grupo PSHC, $23(25,3 \%)$ ao grupo HGE e $36(39,5 \%)$ ao grupo PE. Entre as SHG, os grupos com HC e PSHC apresentaram idade materna estatisticamente superior ao grupo $\mathrm{NH}(p=0,017)$, e a menor idade foi observada nos casos com PE.

Entre as formas das SHG a mais freqüente foi a HGE, seguida pela $P E$, de acordo com a frequência demonstrada por outros estudos ${ }^{(5,12)}$. A HC foi observada em $5,1 \%$ dos casos analisados e a PSHC em $6,8 \%$, semelhante aos relatos de outros trabalhos, em que a freqüência de $\mathrm{HC}$ esteve entre $1 \%$ e $5 \%$. A HC está associada ao aumento de risco para mortalidade materna e fetal, principalmente quando a PSHC se desenvolve ${ }^{(13)}$.

A freqüência das SHG é maior em pacientes com idade acima de 30 anos $^{(14)}$. Em nosso dados, a idade materna maior foi associada ao desenvolvimento de 
formas específicas de SHG, como a HC e a PSHC, provavelmente devido ao progressivo dano endotelial que ocorre com o envelhecimento ${ }^{(12,14)}$. A PE isolada foi associada à menor idade materna. Estudos demonstram que a PE é mais freqüente e desenvolve maiores complicações nos extremos de idade materna. Em gestantes com idade inferior a 20 anos, ela ocorre principalmente associada a primiparidade, sugerindo a participação de fatores imunológicos na sua etiopatogênese, enquanto em faixas etárias maiores, a HC parece ser o principal fator predisponente $^{(12)}$.

Quanto à paridade, a freqüência das SHG foi superior entre as pacientes multíparas. Entre os tipos de SHG, nenhuma paciente com HC era primípara e apenas duas do grupo PSHC tiveram a primeira gestação $(p=0,099)$. A freqüência de cesarianas foi significativamente superior nas pacientes com SHG $(p<0,001)$.

Houve predomínio de pacientes multíparas no grupo SHG, principalmente nos casos com $\mathrm{HC}$ e PSHC. Estes dados são diferentes dos observados por outros autores, onde a hipertensão durante a gestação, principalmente a $\mathrm{HG}$ e a PE, esteve relacionada à primiparidade. Para alguns autores, a PE é considerada predominante, mas não exclusiva da primiparidade. Nas primíparas, as SHG são essencialmente primárias, “induzidas” pela gestação. Nas multíparas, as SHG representam uma doença hipertensiva oculta que é "revelada" pela gestação. Em relação aos grupos com HC e PSHC, a associação com a multiparidade pode ser comprovada por outros estudos $^{(13)}$. Outra hipótese para a freqüência superior de hipertensão entre as multíparas seria a gestação com parceiros diferentes, sugerindo que a doença seria causada pela primipaternidade, e não primiparidade. Uma exposição prolongada aos antígenos fetais de uma gestação prévia, ou paternos, por um longo período de contato com o esperma do parceiro, pode proteger contra a PE em uma gestação subseqüente, sugerindo a influência de fatores genéticos e imunológicos, fetais e/ou paternos, na etiologia da $\mathrm{PE}^{(15)}$.

O parto cesariana foi mais freqüente nas pacientes com SHG. Embora existam várias drogas anti-hipertensivas para uso durante a gestação(16) a interrupção da gestação parece ser a única forma de impedir a evolução das alterações fetais e maternas relacionadas à hipertensão ${ }^{(12,14)}$. Estudos demonstram a maior freqüência de cesarianas em gestantes com hipertensão durante a gestação(14) sendo a interrupção da gestação a responsável por cerca de $15 \%$ dos partos pré-termo, principalmente em casos de HC graves $^{(13)}$. Estes dados estão de acordo com o presente estudo, onde houve redução da IG nas pacientes com PE e PSHC, submetidas mais freqüentemente a interrupção precoce da gestação através da cesariana.

A menor IG e os menores índices de Apgar foram observados nos casos com PE e PSHC. Não houve diferença significativa entre os pesos dos recém-nascidos nos grupos estudados. Houve diferença significativa do peso dos recém-nascidos a termo do grupo SHG quando comparados aos valores populacionais, apresentando valores superiores ao esperado. Esta diferença não foi observada quando os grupos de SHG foram analisados separadamente. Recém-nascidos prematuros de pacientes com SHG apresentaram peso inferior, principalmente nos grupos PE e PSHC. Entre os prematuros o menor peso placentário foi observado nas pacientes com SHG, principalmente nos casos com PE, com diferença significativa. O grupo SHG, principalmente com PE e PSHC, apresentou peso placentário significativamente inferior ao esperado para a população (Tabela 1). 
Tabela 1: Comparação do peso dos recém-nascidos, peso da placenta e da idade gestacional (IG) entre os grupos Não Hipertensas ( $\mathrm{NH}$ ) e Síndromes Hipertensivas da Gestação (SHG), avaliados na Universidade Federal do Triângulo Mineiro, Uberaba, 2002.

\begin{tabular}{|c|c|c|c|c|c|c|}
\hline \multirow[t]{2}{*}{ SHG } & n (\%) & IG (sem) & Peso dos RN (g) & Peso RN/IG (g) & $\mathrm{PP}(\mathrm{g})$ & $P P / I G(g)$ \\
\hline & & \multicolumn{5}{|c|}{$X \pm$ DP ou Mediana (Mínimo - Máximo) } \\
\hline \multicolumn{7}{|l|}{ Termo } \\
\hline $\mathrm{NH}$ & $18(30,5)$ & $39,6 \pm 0,9$ & $3264,4 \pm 428,2$ & 3163 & $461,6 \pm 102,5$ & 490 \\
\hline HG & $17(28,8)$ & $39,2 \pm 1,6$ & $3230,3 \pm 583,2$ & 3163 & $470,9 \pm 114,8$ & 490 \\
\hline PE & $17(28,8)$ & $38,6 \pm 1,7$ & $3161,8 \pm 577,7$ & 2920 & $495,9 \pm 118,4$ & 480 \\
\hline $\mathrm{HC}$ & $4(6,8)$ & $39,9 \pm 2,4$ & $2921,3 \pm 397,8$ & 3163 & $495,0 \pm 91,5$ & 490 \\
\hline PSHC & $3(5,1)$ & $38,8 \pm 1,1$ & $3273,3 \pm 609,6$ & 2920 & $500,0 \pm 104,4$ & 480 \\
\hline \multirow[t]{2}{*}{ Total } & $59(100)$ & & & & & \\
\hline & $\mathbf{p}$ & 0,29 & 0,812 & $>0,05$ & 0,889 & $>0,05$ \\
\hline \multicolumn{7}{|l|}{ Prematuro } \\
\hline HG & $6(18,8)$ & $35,7(34,5-36,1)$ & $2521,7 \pm 415,0$ & 2165 & $450,3 \pm 82,3$ & 435 \\
\hline PE & $19(59,3)$ & $31,7(30,0-34,1)$ & $1648,7 \pm 713,0$ & 1663 & $300,4 \pm 120,4^{1}$ & $380^{2}$ \\
\hline $\mathrm{HC}$ & $2(6,3)$ & $31,2(26,4-36,0)$ & $1963,5 \pm 1395,1$ & 1488 & $350,0 \pm 99,0$ & 360 \\
\hline PSHC & $5(15,6)$ & $33,6(31,8-34,4)$ & $1683,0 \pm 348,7$ & 1838 & $326,0 \pm 51,3^{1}$ & $400^{2}$ \\
\hline \multirow[t]{2}{*}{ Total } & $32(100)$ & & & & & \\
\hline & $\mathbf{p}$ & 0,160 & 0,063 & $>0,05$ & 0,044 & $<0,05$ \\
\hline $\begin{array}{l}\text { HG: Hiperte } \\
\text { HC: Hiperter } \\
\text { PE: Pré-eclâ }\end{array}$ & $\begin{array}{l}\text { são gestacion } \\
\text { são crônica; } \\
\text { psia; }\end{array}$ & & $\begin{array}{l}\text { PSHC } \\
\text { Peso } \\
1,2 \mathrm{Te}\end{array}$ & $\begin{array}{l}\text { Pré-eclâmpsia sok } \\
\text { sperado para a IG } \\
\text { te de Dunn: } p<0,0\end{array}$ & eposta a HC; & \\
\hline
\end{tabular}

Em nossos dados, pacientes com SHG apresentaram redução do peso placentário, e peso menor do que o esperado para a população nos casos com PE e PSHC. Na gestação, mudanças fisiológicas e alterações patológicas podem coincidir com alterações do crescimento fetal. Estudos experimentais demonstram que a placenta é a primeira a sofrer as conseqüências da redução do fluxo sangüíneo uteroplacentário, sendo evidenciadas pelo decréscimo em suas medidas. A restrição de crescimento intra-uterino inclui a alteração de outros fatores além da hipóxia, como a redução do suprimento de nutrientes ou redução da síntese de fatores de crescimento. Dessa forma, a magnitude da redução da massa placentária pode refletir o grau de comprometimento do fluxo uteroplacentário, influenciando tardiamente no peso fetal ${ }^{(17)}$. Portanto, - exame da placenta pode contribuir para diagnosticar alterações da gestação que não atingiram o feto, mas deixaram suas evidências na placenta, detectadas mesmo na macroscopia.

$O$ peso fetal foi inferior nos grupos com SHG, principalmente nos casos com PE e HC, embora quando comparados com os pesos da população esperados para a mesma idade gestacional não tenham apresentado diferença significativa. $O$ peso ao nascimento é determinado pela duração da gestação e pela restrição de crescimento intrauterino. O vaso espasmo e o decréscimo do volume intravascular possuem um importante papel no crescimento fetal e pode explicar a maior incidência de restrição do crescimento fetal nas formas graves de SHG, como a PE e PSHC. A PE é relacionada a maiores riscos agudos para a mãe e o feto, enquanto a HC aumentaria os riscos de restrição de crescimento intra-uterino, com efeitos agudos maiores quando associada a PSHC ${ }^{(12)}$. O menor peso, a menor IG e os menores índices de Apgar no primeiro e no quinto minuto foram observados nos casos de PE e PSHC. O Índice de Apgar é considerado o melhor valor preditivo de intercorrências a longo tempo ${ }^{(18)}$, e associado à menor idade gestacional encontrada, demonstram que a gravidade clínica das SHG foram determinantes para o tempo de interrupção da gestação e o desenvolvimento do sofrimento fetal agudo.

Pela avaliação pela Hematoxilina e Eosina, nas gestações a termo, os casos com PE e HC apresentaram maior número de vasos nas vilosidades terminais $(p<0,001)$. Nos prematuros, o maior número de vasos foi observado nos casos com PE $(p<0,001)$ (Tabela 2). 
Tabela 2: Comparação do numero de vasos das vilosidades terminais avaliadas através da coloração pela Hematoxilina e Eosina nos casos com Síndromes Hipertensivas da Gestação (SHG), avaliados na Universidade Federal do Triângulo Mineiro, Uberaba, 2002.

\begin{tabular}{|c|c|c|}
\hline SHG & n (\%) & Número de vasos nas vilosidades terminais ( $\mathrm{HE}$ ) \\
\hline Termo & & Mediana(Mínimo - Máximo) \\
\hline Não Hipertensas & $18(30,5)$ & $7,0(5,0-8,3)$ \\
\hline Hipertensão gestacional & $17(28,8)$ & $6,0(5,0-8,0)^{1,2}$ \\
\hline Pré-eclâmpsia (PE) & $17(28,8)$ & $7,0(6,0-9,0)^{1}$ \\
\hline Hipertensão Crônica (HC) & $4(6,8)$ & $8.0(6,0-10,5)^{2}$ \\
\hline PE sobreposta a HC & $3(5,1)$ & $6,0(5,0-8,0)$ \\
\hline Total & $59(100)$ & \\
\hline $\mathbf{p}$ & & $<0,001$ \\
\hline \multicolumn{3}{|l|}{ Prematuros } \\
\hline Hipertensão gestacional & $6(18,8)$ & $6,0(5,0-8,0)^{3}$ \\
\hline Pré-eclâmpsia & $19(59,4)$ & $7,0(5,0-9,0)^{3}$ \\
\hline Hipertensão Crônica & $2(6,3)$ & $6,0(5,0-7,0)$ \\
\hline PE sobreposta a $\mathrm{CH}$ & $5(15,5)$ & $6.0(5,0-8,0)$ \\
\hline Total & $32(100)$ & \\
\hline $\mathbf{p}$ & & $<0,001$ \\
\hline
\end{tabular}

1,2,3Teste de Dunn: $p<0,05$

Entre as SHG, o maior número de vasos das vilosidades foi observado nos casos com $\mathrm{HC}$, em gestações a termo e prematuros. Existem estudos que demonstram 0 aumento de fatores de crescimento, como o VEGF, estimulando a angiogênese nos casos de hipóxia ${ }^{(19)}$. Outra explicação para o aumento do número de vasos durante a hipertensão consiste em uma falência no remodelamento das arteríolas espiraladas, reduzindo - fluxo sanguíneo placentário, promovendo a alteração na diferenciação e proliferação do trofoblasto, responsável pelo aumento de nós sinciciais, distensão e aumento dos capilares intravilositários, considerado um marcador placentário de hipofluxo sanguíneo ${ }^{(8)}$. As pacientes com HC, por serem expostas a um maior tempo de alterações hemodinâmicas, e possivelmente, apresentarem comprometimento da vascularização uterina, apresentaram maiores alterações nos vasos das vilosidades terminais, representadas pela maior proliferação vascular.

Nos casos com PSHC e intercorrências perinatais, um menor número de vasos foi observado. Com a manutenção da hipóxia placentária ocorre uma inversão do padrão de proliferação vascular. O maior comprometimento fetal e o desenvolvimento vilositário anormal levam a redução da extração de oxigênio pelo feto, resultando em hiperóxia placentária, reduzindo os estímulos para angiogênese, resultando em colapso dos capilares ${ }^{(19)}$. Nos casos com PSHC e natimortalidade, o comprometimento do fluxo sangüíneo uteroplacentário pode ter sido mais grave, resultando em diminuição da capacidade de extração de oxigênio pelo feto e levando ao aumento da concentração de oxigênio placentário no espaço interviloso, e justificando o menor número de vasos encontrados nestes casos.

Através da imunohistoquímica, não houve diferença entre o número de vasos das vilosidades tronco entre os grupos NH e SHG. Nas vilosidades intermediárias, o número de vasos foi maior nos casos com hipertensão, na região do córion e da decídua dos casos com gestação a termo. Entre os prematuros, o maior número de vasos foi observado na porção intermediária da placenta das $\mathrm{NH}$. Entre os tipos de SHG, o número de vasos foi maior nos casos com HGE e PE nas três porções da placenta. Entre os prematuros, o menor número de vasos foi observado na porção intermediária dos casos com PSHC (Tabela $3)$. 
Tabela 3: Comparação do número de vasos imunomarcados com CD31 das vilosidades intermediárias nas regiões próximas ao córion, intermediária e da decídua entre os grupos de Não Hipertensas (NH) e síndromes hipertensivas da gestação (SHG), avaliados na Universidade Federal do Triângulo Mineiro, Uberaba, 2002.

\begin{tabular}{|c|c|c|c|}
\hline $\begin{array}{l}\text { Parte da } \\
\text { placenta }\end{array}$ & SHG & $\begin{array}{l}\text { Gestação a termo } \\
\text { Número de vasos }\end{array}$ & $\begin{array}{c}\text { Prematuros } \\
\text { Número de vasos }\end{array}$ \\
\hline & & Mediana (Mínimo - Máximo) & Mediana (Mínimo - Máximo) ou X \pm DP \\
\hline \multirow{6}{*}{ Córion } & HG & $14,0(12,0-21,0)$ & $8,0(6,0-10,0)$ \\
\hline & $\mathrm{HC}$ & $10,0(8,0-17,0)$ & $8,0(5,8-13,5)$ \\
\hline & PE & $11,5(6,5-14,5)$ & $11,0(8,0-16,3)$ \\
\hline & PE sobreposta a HC & $8,0(4,0-12,3)$ & $11,5(8,0-16,0)$ \\
\hline & $\mathrm{NH}$ & $4,5(4,0-5,0)$ & $10,0(5,3-14,8)$ \\
\hline & $\mathbf{p}$ & $<0,001$ & 0,06 \\
\hline \multirow{6}{*}{ Intermediária } & HG & $11,5(7,0-19,0)$ & $9,0(7,0-11,0)$ \\
\hline & $\mathrm{HC}$ & $9,0(7,0-11,8)$ & $9,0(6,0-11,0)$ \\
\hline & PE & $17,0(8,0-25,0)$ & $11,0(7,0-15,5)$ \\
\hline & PE sobreposta a HC & $6,0(5,0-8,0)$ & $6,0(4,0-8,0)$ \\
\hline & $\mathrm{NH}$ & $9,0(5,8-13,3)$ & $15,0(11,0-23,3)$ \\
\hline & $\mathbf{p}$ & 0,02 & 0,002 \\
\hline \multirow{6}{*}{ Decídua } & HG & $19,0(10,0-31,3)$ & $10,4 \pm 5,6$ \\
\hline & $\mathrm{HC}$ & $10,5(6,5-15,0)$ & $10,2 \pm 4,1$ \\
\hline & PE & $14,0(7,0-22,5)$ & $10,7 \pm 7,1$ \\
\hline & PE sobreposta a HC & $10,0(7,0-19,0)$ & $10,1 \pm 5,8$ \\
\hline & $\mathrm{NH}$ & $6,0(5,0-7,8)$ & $10,7 \pm 4,9$ \\
\hline & $\mathbf{p}$ & 0,022 & 0,999 \\
\hline
\end{tabular}

HG: Hipertensão Gestacional;

HC: Hipertensão Crônica;

PE: Pré-eclampsia.

Nas vilosidades terminais, os casos de gestações a termo apresentaram menor número de vasos nos casos com SHG quando comparado às $\mathrm{NH}$ em todas as regiões da placenta. Entre os prematuros a distribuição foi inversa, sendo maior nos casos com SHG. O número de vasos nas vilosidades terminais foi menor nos casos com PSHC e maiores nos casos com PE e HGE (Tabela 4).

Tabela 4: Comparação do número de vasos imunomarcados com CD31 das vilosidades terminais nas regiões próximas ao córion, intermediária e da decídua entre os grupos de síndromes hipertensivas da gestação (SHG), avaliados na Universidade Federal do Triângulo Mineiro, Uberaba/MG, 2002.

\begin{tabular}{|c|c|c|c|}
\hline Parte da placenta & SHG & $\begin{array}{l}\text { Gestação a termo } \\
\text { Número de vasos }\end{array}$ & $\begin{array}{c}\text { Prematuros } \\
\text { Número de vasos }\end{array}$ \\
\hline & & \multicolumn{2}{|c|}{ Mediana (Mínimo - Máximo) } \\
\hline \multirow{6}{*}{ Córion } & HG & $4,0(3,0-6,0)$ & $5,0(4,0-6,0)$ \\
\hline & $\mathrm{HC}$ & $5,0(3,0-6,0)$ & $4,0(3,0-5,0)$ \\
\hline & PE & $5,0(4,0-6,7)$ & $5,0(4,0-7,0)$ \\
\hline & PE sobreposta a HC & $3,0(3,0-5,0)$ & $4,0(3,0-6,0)$ \\
\hline & Não Hipertensas & $5,0(4,0-6,0)$ & $4,0(3,0-5,0)$ \\
\hline & $\mathbf{p}$ & $<0,001$ & $<0,001$ \\
\hline \multirow{6}{*}{ Intermediária } & HG & $5,0(4,0-7,0)$ & $5,0(4,0-7,0)$ \\
\hline & $\mathrm{HC}$ & $5,0(3,0-7,0)$ & $3,0(3,0-5,0)$ \\
\hline & PE & $5,0(3,0-7,0)$ & $5,0(4,0-7,0)$ \\
\hline & PE sobreposta a HC & $4,0(3,0-4,0)$ & $4,0(4,0-6,0)$ \\
\hline & Não Hipertensas & $5,0(4,0-7,0)$ & $4,0(3,0-5,0)$ \\
\hline & $\mathbf{p}$ & $<0,001$ & $<0,001$ \\
\hline \multirow{6}{*}{ Decídua } & HG & $5,0(4,0-7,0)$ & $4,0(3,0-5,0)$ \\
\hline & $\mathrm{HC}$ & $4,0(3,0-6,0)$ & $4,0(3,0-6,0)$ \\
\hline & PE & $5,0(4,0-7,0)$ & $4,0(3,0-6,0)$ \\
\hline & PE sobreposta a HC & $4,0(3,0-5,0)$ & $5,0(4,0-7,0)$ \\
\hline & Não Hipertensas & $5,0(4,0-7,0)$ & $4,0(3,0-5,0)$ \\
\hline & $\mathbf{p}$ & $<0,001$ & $<0,001$ \\
\hline
\end{tabular}

HG: Hipertensão Gestacional; HC: Hipertensão Crônica; 
Para comprovar a proliferação de vasos nas vilosidades coriônicas foi utilizado o anticorpo antiCD31 para imunomarcação do endotélio vascular, que demonstrou uma variação do número de vasos nos diversos compartimentos placentários, nas vilosidades intermediárias e terminais, demonstrando relação com a gravidade da síndrome hipertensiva. Os casos menos graves e crônicos para a placenta, como a PE e a HGE, foram associados ao aumento do número de vasos, que freqüentemente foi acompanhado da ampliação da área das vilosidades. Nos casos mais graves, como na PSHC, houve redução do número de vasos e, conseqüentemente da área das vilosidades, dados também observados com a coloração pela Hematoxilina e Eosina, comprovando o desequilíbrio da homeostasia placentária em condições de hipóxia. Os critérios diagnósticos e classificação das SHG são baseados em informações laboratoriais. Entretanto, apenas a informações clínicas podem não ser suficientes para responder questões como se os tipos de SHG representam diferentes estágios de uma mesma doença, ou se cada tipo de SHG envolve mecanismos diferentes de desenvolvimento ${ }^{(20)}$.

\section{CONCLUSÃO}

Este estudo sugere que embora existam diferentes intervenções para os tipos de SHG, o mecanismo final que leva às alterações microscópicas dos vasos placentários é o mesmo, e as alterações representam apenas diferentes intensidades relacionadas à gravidade do quadro clínico. Em conclusão, este estudo demonstrou uma variação de parâmetros classicamente associados às SHG em relação aos seus tipos, contribuindo para caracterização demográfica, morfológica e morfométrica dos diferentes tipos de SHG e proporcionando uma maior correspondência entre os achados do exame anatomopatológico placentário e a clínica apresentada durante a gestação. Estes dados podem facilitar o diagnóstico médico e de enfermagem, bem como o planejamento da assistência de todos os profissionais envolvidos no atendimento do recém-nascido, da criança, e da mãe, mesmo fora do período puerperal.

\section{REFERÊNCI AS}

1. Corrêa RRM, Belela ASC, Ferraz MLF, Paschoin MC, Teixeira VPA, Reis MA et al. O espessamento da membrana basal do epitélio amniótico e as síndromes hipertensivas da gestação. Revista Médica de Minas Gerais. 2003; 14(4): 221-5.

2. Van Oppenraaij RH, Jauniaux E, Christiansen OB, Horcajadas JA, Farquharson RG, Exalto $\mathrm{N}$ et al. Predicting adverse obstetric outcome after early pregnancy events and complications: a review. Human Reproduction. 2009; 15(4):409-21.
3. Teasdale, F. Histomorphometry of the human placenta in maternal preeclampsia. Am J Obstet Gynecol. 1985; 152(1): 25-31.

4. Yucesoy G, Ozkan S, Bodur H, Tan T, Caliskan E, Vural B et al. Maternal and perinatal outcome in pregnancies complicated with hypertensive disorder of pregnancy: a seven year experience of a tertiary care center. Arch Gynecol Obstet. 2005;273(1):43-9.

5. Gifford RW. Report of the National High Blood Pressure Education Program Working Group on High Blood Pressure in Pregnancy. Am J Obstet Gynecol. 2000; 183(1): 1-22.

6. Zhou Y, McMaster M, Woo K, Janatpour M, Perry J, Karpanen $\mathrm{T}$ et al. Vascular endothelial growth factor ligands and receptors that regulate human cytotrophoblast survival are dysregulated in severe preeclampsia and hemolysis, elevated liver enzymes, and low platelets syndrome. Am J Pathol. 2002; 160(4): 1405-23.

7. Norwitz ER. Defective implantation and placentation: laying the blueprint for pregnancy complications. Reprod Biomed Online. 2006; 13(4): 591-9.

8. Kingdom JC, Kaufmann P. Oxygen and placental villous development: origins of fetal hypoxia. Placenta. 1997; 18(8):613-21.

9. Mayhew TM, Burton GJ. Stereology and its impact on our understanding of human placental functional morphology. Microsc Res Tech. 1997;38(1-2): 195205.

10. Gruenwald P, Minh HN. Evaluation of body and organ weights in perinatal pathology. II. Weight of body and placenta of surviving and of autopsied infants. Am J Obstet Gynecol. 1961;82:312-19.

11. Hawfield A, Freedman BI. Pre-eclampsia: the pivotal role of the placenta in its pathophysiology and markers for early detection. Therapeutic Advances in Cardiovascular Disease. 2009; 3(1):65-73.

12. Roberts JM, Pearson GD, Cutler JA, Lindheimer MD. Summary of the NHLBI Working Group on Research on Hypertension During Pregnancy. Hypertens Pregnancy. 2003;22(2): 109-27.

13. Vigil-De Gracia P, Lasso M, Montufar-Rueda C. Perinatal outcome in women with severe chronic hypertension during the second half of pregnancy. International Journal of Gynecololgy e Obstetrics. 2004; 85(2): 139-44.

14. Coelho TM, Martins MG, Viana E, Mesquita MRS, Camano L, Sass N. Proteinúria nas síndromes hipertensivas da gestação: prognóstico materno e perinatal. Rev Assoc Med Bras. 2004;50(2): 207-13.

15. Saftlas AF, Levine RJ, Klebanoff MA, Martz K, Ewell MG, Morris $C D$ et al. Abortion, changed paternity, and risk of preeclampsia in nulliparous women. Am J Epidemiol. 2003; 157(12) : 1108- 14.

16. Rosenthal T, Oparil S. The effect of antihypertensive drugs on the fetus. Journal of 
Human Hypertension. 2002; 16(5): 293-8.

17. Buchbinder A. Adverse perinatal outcomes are significantly higher in severe gestational hypertension than in mild preeclampsia. Am J Obstet Gynecol. 2002; 186(1): 66-71.

18. Senesi LG, Tristão EG, Andrade RP, Krajden ML, Oliveira Júnior FC, Nascimento DJ. Morbidade e mortalidade neonatais relacionadas à idade materna igual ou superior a 35 anos, segundo a paridade. Rev. Bras. Ginecol. Obstet. 2004;26(6):477-82.

19. Burton GJ. On oxygen and placental villous development: origins of fetal hypoxia. Placenta. $1997 ; 18(8)$ : 625-6.

20. Medina Lomeli JM, Medina Castro N. Differences and similarities of preeclampsia and gestational hypertension. Ginecología y Obstetricia de México. 2005; 73(10): 48-53.

Artigo recebido em 25.05.08.

Aprovado para publicação em 06.05.09.

Artigo publicado em 30.09.09. 